\title{
Loading of Drug-Polymer Matrices in Microreservoirs for Oral Drug Delivery
}

\author{
Petersen, Ritika Singh; Keller, Stephan Sylvest; Boisen, Anja
}

Published in:

Macromolecular Materials \& Engineering

Link to article, DOI:

10.1002/mame.201600366

Publication date:

2017

Document Version

Peer reviewed version

Link back to DTU Orbit

Citation (APA):

Petersen, R. S., Keller, S. S., \& Boisen, A. (2017). Loading of Drug-Polymer Matrices in Microreservoirs for Oral Drug Delivery. Macromolecular Materials \& Engineering, 302(3), [1600366].

https://doi.org/10.1002/mame.201600366

\section{General rights}

Copyright and moral rights for the publications made accessible in the public portal are retained by the authors and/or other copyright owners and it is a condition of accessing publications that users recognise and abide by the legal requirements associated with these rights.

- Users may download and print one copy of any publication from the public portal for the purpose of private study or research.

- You may not further distribute the material or use it for any profit-making activity or commercial gain

- You may freely distribute the URL identifying the publication in the public portal

If you believe that this document breaches copyright please contact us providing details, and we will remove access to the work immediately and investigate your claim 


\section{Loading of drug-polymer matrices in microreservoirs for oral drug delivery}

Ritika Singh Petersen, Stephan Sylvest Keller, and Anja Boisen

Department of Micro- and Nanotechnology, Technical University of Denmark, Building 345E, DK2800 Kongens Lyngby, Denmark

e-mail: risi@nanotech.dtu.dk

Keywords: hot punching, microcontainers, biopolymer, drug delivery, drug-polymer matrix

The oral route remains the preferred method for drug delivery due to high patient compliance and administration without the need for health care professionals. However, conventional dosage forms such as tablets and capsules are facing a number of limitations. Among others, the short retention time in the intestine is a challenge, in particular for drugs with low solubility and low permeability through the intestinal mucosa. This means that a considerable amount of the drug passes through the gastrointestinal tract (GIT) without being able to reach its target. Furthermore, degradation of the active pharmaceutical ingredient (API) has to be considered specifically in the case of protein or peptide based drugs. Microfabricated reservoir based devices have been proposed as advanced oral drug delivery system to potentially address some of these issues by preventing the degradation of drug and allowing unidirectional release in the GIT. Recently, first in vivo studies have shown promising results for prolonged release and enhanced bioavailability of drugs . ${ }^{[1-4]}$ The microreservoirs have conventionally been fabricated with $\mathrm{Si}$ and photoresists such as SU-8 and poly(methyl methacrylate) (PMMA) using standard micro-electro-mechanical systems (MEMS) fabrication technologies. ${ }^{[5,6]}$ Gradually, the focus has shifted to fabricating drug reservoirs in biodegradable polymers such as poly(lactic-co-glycolic acid) (PLGA). ${ }^{[7,8]}$ Once these microreservoirs are fabricated, drug loading imposes a major challenge. The loaded drug is preferentially embedded in a polymer matrix for stabilization and to tailor the release properties. 
The polymer matrices can be hydrophobic, slow-degrading biodegradable polymers such as poly-1lactic acid (PLLA) or poly- $\varepsilon$-caprolactone (PCL) for sustained drug release. ${ }^{[9]}$ Alternatively, the drug matrices can be hydrophilic polymers or their gel forms such as poly acrylic acid (PAA) and poly vinyl pyrrolidone (PVP) for increased drug solubility and bioavailability. ${ }^{[10,11]}$ In the past, microreservoirs have been loaded with drug-polymer matrices by using photolithography, microinjection and ink-jet printing. ${ }^{[12-14]}$ Supercritical $\mathrm{CO}_{2}(\mathrm{scCO} 2)$ has been introduced as a method to load drug in microreservoirs previously filled with polymer matrix by ink-jet printing. ${ }^{[15]}$ Although aforementioned loading techniques provide good control on the amount of loaded drug, they suffer from some disadvantages. Photolithography typically uses UV light which can degrade the active pharmaceutical ingredients (API) and it is challenging to handle high molecular weight polymers and drugs. ScCO2 impregnation has some limitations because of limited solubility of APIs. Furthermore, the structural stability of microreservoirs fabricated in biodegradable polymers such as PLLA can be affected in $\mathrm{scCO}_{2}$. The common limitation with all these loading techniques is that they are relatively slow processes. Alternatively, microcontact printing has been developed as a fast process to fill a large number of microreservoirs in parallel. ${ }^{[16]}$ However, this technique requires fabrication of a polydimethylsiloxane (PDMS) stamp which is mechanically and chemically not sufficiently stable to perform a large number of loading runs. ${ }^{[17]}$ Microcontact printing also requires a set-up to align the stamp precisely to the microreservoirs. ${ }^{[16]}$

In this paper, a new fast method for parallel loading of reservoir based microdevices called microcontainers is proposed and demonstrated. Each microcontainer includes a reservoir surrounded by a thin wall.The containers are fabricated in the negative epoxy photoresist SU-8 and in biopolymer PLLA to demonstrate the versatility of the drug loading method. SU-8 microcontainers are fabricated using two-steps of photolithography on a fluorocarbon coated $\mathrm{Si}$ carrier substrate. ${ }^{[18]}$ PLLA microcontainers are fabricated using hot punching and transfer to a water 
soluble sacrificial layer deposited on $\mathrm{Si}$ substrate. ${ }^{[19]}$ The containers are produced as arrays on a fluorocarbon or sacrificial layer respectively to allow final release from the Si substrate. After the fabrication of microcontainers, the reservoirs of the containers are loaded with drug-polymer matrix using hot punching. Figure 1 illustrates the overall concept of drug loading in the two types of microcontainers.

Hot punching is a combination of hot embossing and mechanical punching processes. ${ }^{[19-21]}$ Hot embossing is the process of achieving micropatterns in a viscoelastic polymer film placed on a carrier substrate when a mold is embossed into the film, through the application of external pressure and temperature. Mechanical punching process allows to produce through holes in a polymer film where the punched out polymer structures are basically the by-product of the process. ${ }^{[22]}$ During the hot punching process a mold is embossed in a polymer film at elevated temperature, separating the embossed polymeric microstructures from the rest of the film. Two conditions need to be considered during hot punching: i) either the mold or the substrate carrying the polymer film should locally display some elastic deformation upon application of the pressure. This provides the force required to rupture the polymer film and to allow mold and carrier substrate to come in physical contact and ii) the thickness of the polymer film has to be sufficiently high to fill the cavities of the mold but low enough to allow contact of the mold with the carrier substrate during the application of pressure. Once these conditions are satisfied, the hot punching process promotes disruption of the residual layer inherently present after a typical hot embossing process and thus leads to formation of discrete 3D microstructures.

For the drug loading process of the microcontainers, the arrays of microcontainers on the substrate are considered as the mold (Figure 1.A.1 and 1.B.1). This microcontainer mold punches the drugpolymer film spin coated on a substrate by applying temperature and pressure. A third condition 
needs to be fulfilled to successfully load the microcontainers: iii) the 3D drug-polymer microstructures that are punched out by the microcontainermold during the hot punching process, have to remain in the microcontainers after demolding, thereby loading the containers. To ensure this, the work of adhesion $W_{p m}$ between mold and drug-polymer film should be higher than the work of adhesion $W_{p s}$ between the drug-polymer film and the carrier substrate. For the proof-ofconcept, we selected the drug-polymer combination of furosemide (F), a loop diuretic drug, and biodegradable poly- $\varepsilon$-caprolactone (PCL) polymer as a model system. ${ }^{[23]}$ The drug-polymer film (FPCL) is fabricated by spin coating a homogenous F-PCL solution on the substrate. The F-PCL film is punched by the SU-8 and PLLA microcontainermold during the hot punching process.

In order to punch F-PCL film using thermally non-deformable rigid SU-8 microcontainers, the Si substrate is coated with PDMS before spin coating F-PCL solution (Figure 1.A.1). The elastically deformable PDMS film allows the contact of the mold and the substrate. ${ }^{[18]}$ For the punching of FPCL film by thermally deformable PLLA microcontainers, F-PCL solution is spin coated directly on Si without any additional PDMS layer (Figure 1.B.1). Here, the elastically deformable microcontainers themselves promote rupture of the residual layer. In order to fulfill the second condition for hot punching, the required thickness of the F-PCL film is calculated based on the dimensions of the microcontainers (Supporting information). The thickness of the F-PCL film should be less than or equal to the thickness required to completely fill the microcontainer reservoirs without leaving a residual film. The critical dimensions of the containers are summarized in Table 1 and illustrated in Figure. B of Supporting information. The calculated optimal thickness of the F-PCL film for loading of SU-8 containers is $48 \mu \mathrm{m}$ and for PLLA microcontainers it is 70 $\mu \mathrm{m}$. 
During the punching process, the F-PCL film is punched along the walls of the microcontainers through the application of pressure and temperature. The results are summarized in Figure 2. The hot punching of F-PCL film by SU-8 microcontainers is performed at a temperature of $65^{\circ} \mathrm{C}$ and a pressure of 1 bar. The temperature is applied on both plates of the embossing equipment in contact with the microcontainermold and the F-PCL sample. When the SU-8 containers punch the F-PCL film lying on PDMS (Figure 1.A.2), $W_{p m}>W_{p s}$ due to the hydrophobic properties of PDMS. Hence, the punched out F-PCL microstructures remain in the SU-8 reservoir and the SU-8 microcontainers are loaded (Figure 1.A.3, 2.A, 2.B and 2.C). Complete filling of reservoirs is achieved by spin coating F-PCL films with a thickness of around $46 \pm 3 \mu \mathrm{m}$, which is in good agreement with the calculated film thickness of $48 \mu \mathrm{m}$.

When the temperature of $65^{\circ} \mathrm{C}$ is applied to both plates of the embossing equipment for loading of F-PCL in PLLA microcontainers, the thermally deformable PLLA containers lose their structural integrity and are flattened. The glass transition temperature of PLLA is between $65-70{ }^{\circ} \mathrm{C}^{[24]}$, and punching at a temperature lower than $65{ }^{\circ} \mathrm{C}$ results in poor yield of loading.Consequently a temperature of $75{ }^{\circ} \mathrm{C}$ is only applied to the plate in contact with the F-PCL sample while the plate with the PLLA containers remains at ambient temperature. Furthermore, the applied pressure is lowered to 0.5 bar in order to achieve good yield of loading without affecting the integrity of the containers(Figure 1.B.2). The F-PCL polymer film at $75^{\circ} \mathrm{C}$, locally heats the PLLA upon contact during hot punching and blends with it at the interface. ${ }^{[24,25]}$ This results in sufficiently high $W_{p m}$ to ensure that the punched out F-PCL structures remain in the PLLA microcontainers after demolding, thereby loading them (Figure 1.B.3, 2. D, 2.E and 2.F).

Optical profiler measurements show a filling height of $61 \mu \mathrm{m}$ and indicate that the PLLA containers remain structurally intact (Figure 2.G and H). Successful loading is achieved even for a F-PCL 
film thickness as low as $43 \pm 3 \mu \mathrm{m}$ which is considerably below the calculated optimal value of 70 $\mu \mathrm{m}$. This is explained by the high $\mathrm{W}_{\mathrm{pm}}$ and polymer reflow during punching of the F-PCL film in a molten state. High replicability and yield of $>95 \%$ are observed for the loading of both SU-8 and PLLA microcontainers.

The model system of F-PCL matrix loaded in the microcontainers by hot punching is characterized by performing X-Ray Powder Diffraction (XRPD) and Raman spectroscopy. The furosemide release is studied by microdissolution. For the sake of simplicity, XRPD and Raman data for F-PCL loaded in PLLA microcontainers is presented here. The results confirm that both PCL and furosemide are loaded in the microcontainers. Furosemide is present in polymorph form I which is the most common and the most stable of all the polymorphs due to more efficient crystal packing, higher density and the presence of sulfonamide groups. ${ }^{[23]}$ The spectra taken for the pure furosemide crystals show the same spectra of polymorph form I indicating that the furosemide remains in the most stable form during the punching process as illustrated in Figure 3.A. ${ }^{[24]}$ The sharp furosemide peaks especially at $1600 \mathrm{~cm}^{-1}$ and $1509 \mathrm{~cm}^{-1}$ in raman data and at $25^{\circ} 2 \theta$ in XRPD show that furosemide is present in semi-crystalline form (Figure 3.A and B). ${ }^{[26,}{ }^{27]}$ Microdissolution study depicted in Figure 3.C, illustrates the release of furosemide from the PCL matrix loaded in PLLA microcontainers using hot-punching. The release profile of furosemide shows an initially fast release followed by a slower sustained release. The weight of microcontainers is measured before and after the loading, giving an estimate of the amount of drug present in a container. Based on this, around $80 \%$ of furosemide is released from the PCL matrix after $7 \mathrm{hrs}$ of dissolution. PCL matrix promotes the controlled release of furosemide as no degradation or erosion occurs in the time span of $7 \mathrm{hrs} .^{[28]}$

In conclusion, we here demonstrate hot punching as a new efficient process of loading drugpolymer matrix in microreservoirs. The advantages of the hot punching process for loading of 
micro-reservoir based oral drug delivery devices are: i) it is a versatile process that can be used to load different combinations of drug and polymer; ii) the process is a single-step, parallel process with high throughput; iii) no specialized printing or alignment tools and advanced processing conditions are required which is promising for future scale up; iv) hot punching is a comparatively benign process towards the drug; v) it can be used for filling of high-aspect ratio structures. A potential limitation of the process is the application of elevated temperature, which might affect some APIs. Furthermore, we have shown successful release of drug from microcontainers completely manufactured in biodegradable polymers.

\section{Acknowledgements}

The work presented here has been funded by Danmarks Grundforskningsfonds og Villum Fondens Center for Intelligent Drug Delivery and Sensing Using Microcontainers and Nanomechanics, IDUN (The Danish National Research Foundation: DNRF122, The Velux Foundations: 9301).

\section{Experimental}

\section{Materials}

Standard 4-inch single crystal (SC) silicon wafers are supplied by Okmetic (Vantaa, Finland). Sylgard 184 from Dow Corning is used to prepare PDMS. The prepolymer and the curing agent are mixed in 10:1 ratio. For the experiments, PLLA 2003D grade is purchased from Natureworks (Minnetonka, Minnesota), with $\mathrm{M}_{\mathrm{w}}=126,000 \mathrm{Da}$, determined by size exclusion chromatography measurements. Dichloromethane (DCM) (anhydrous > 99.8\%), poly Acrylic Acid (PAA, average $\mathrm{M}_{\mathrm{w}} \sim 1,800$ ) powder, polyethylene glycol pellets (PEG, average $\mathrm{Mn}=6,000$ ), polycaprolactone pellets (average $\mathrm{M}_{\mathrm{w}} \sim 100,000$ ) and phosphate buffer saline tablets (pH 7.4) are obtained from Sigma Aldrich (Copenhagen, Denmark). Furosemide ( $\geqslant 98 \%$ purity) is supplied by Sigma-Aldrich (St. Louis, MO, USA). SU-8 2075 and SU-8 developer are purchased from Microresist Technology 
GmbH (Berlin, Germany). The chromium mask is designed using L-Edit from Tanner EDA (Monrovia, CA, USA) and supplied by DeltaMask B.V. (GJ Enschede, The Netherlands).

\section{Methods}

\subsection{Loading of microcontainers using hot punching}

First the SU-8 microcontainers and PLLA microcontainers are fabricated using photolithography and hot punching (process B), respectively. For the easy release of the microcontainers, SU-8 fabrication is performed on fluorocarbon coating while PLLA microcontainers are bonded to water soluble sacrificial layer of PAA-PEG. Detailed description of the fabrication process of the containers can be found in the earlier papers [14, 18]. For loading of F-PCL, first a homogenous solution is prepared. $8 \mathrm{~g}$ of PCL and $2 \mathrm{~g}$ of furosemide are dissolved in $40 \mathrm{ml}$ of Acetone and $20 \mathrm{ml}$ of DCM producing a $14.7 \%$ wt. solution. The solution is stirred overnight at room temperature.

In order to load SU-8 microcontainers with F-PCL, F-PCL solution is spin coated at $500 \mathrm{rpm}$ on PDMS deposited Si wafer to give $22 \pm 2 \mu \mathrm{m}$ thick layer. This process is repeated twice to achieve a final thickness of $46 \pm 3 \mu \mathrm{m}$. This spin coated wafer is kept overnight for the evaporation of DCM. Once the DCM solvent evaporates from the PCL layer, it is brought in contact with the SU-8 microcontainers. The punching is done by applying a temperature of $65^{\circ} \mathrm{C}$ and pressure of 1 bar for 30 min to both the upper and lower plates of the embossing tool. The molten F-PCL fills the SU-8 container at this stage. The hot plates are cooled down to $35^{\circ} \mathrm{C}$ and the SU-8 containers are demolded. The punched F-PCL detaches from the surrounding F-PCL film and remains attached to the SU-8 container, thus filling them.

In order to load PLLA microcontainers with F-PCL, the same $14.7 \%$ wt. solution is spin coated twice at $500 \mathrm{rpm}$ as before to achieve $46 \pm 3 \mu \mathrm{m}$ film thickness. Once DCM evaporates, the hot punching of the F-PCL film by the PLLA microcontainers is done by applying a higher temperature 
of $75^{\circ} \mathrm{C}$. This temperature is applied for $30 \mathrm{~min}$ to the plate in contact with the F-PCL sample only in order to protect the structural integrity of PLLA microcontainers. A lower pressure of 0.5 bar required to bring the F-PCL sample and microcontainers in contact is applied and demolding is performed at $35^{\circ} \mathrm{C}$.

\subsection{Scanning electron microscopy and optical profilometry}

The cross-sectional profile measurements of the microcontainers are acquired using confocal mode (20X) in optical profilometry (Sensofar PLu Neox 3D). The samples are characterized using SEM Zeiss Supra $40 \mathrm{VP}$ in variable pressure mode at $8 \mathrm{kV}$ operating voltage and $13 \mathrm{~Pa}$ pressure.

\subsection{Raman spectroscopy and X-Ray diffraction measurements}

Raman spectra are collected from the F-PCL loaded PLLA microcontainers with a DXR Raman microscope (Thermo Scientific, Germany) equipped with a frequency-stabilized single mode diode laser $(780 \mathrm{~nm})$. The laser power is set to $24 \mathrm{~mW}$ at the sample position (center of the container) and the estimated resolution is $2.4-4.4 \mathrm{~cm}^{-1}$. An exposure time of $30 \mathrm{~s}$ and 3 scans are used. The X-ray powder diffraction (XRPD) study is carried out to characterize the physical state of the drug embedded in the PCL matrix loaded in the PLLA microcontainers. All XRPD analyses are performed using an X'Pert PRO X-ray diffractometer (PANalytical, Almelo, The Netherlands; MPD PW3040/60 XRD; Cu KR anode; $\lambda=1.541 \AA ; 45 \mathrm{kV} ; 40 \mathrm{~mA})$. A starting angle of $5^{\circ} 2 \theta$ and an end angle of $35^{\circ} 2 \theta$ are employed for the scans. A scan speed of $0.6565^{\circ} 2 \theta / \mathrm{min}$ and a step size of $0.01313^{\circ} 2 \theta$ are employed. Data are collected using the X'Pert Data Collector software (PANalytical B.V.).

\subsection{In vitro drug dissolution studies}


Dissolution of furosemide from PCL loaded in PLLA microcontainers is measured in $10 \mathrm{ml}$ phosphate buffer media ( $\mathrm{pH} 7.4$ ) at $37.5^{\circ} \mathrm{C}$ using a $\mu$ DISS profiler (Pion) for $7 \mathrm{hrs}$. The media is prepared by dissolving the buffer capsule in $200 \mathrm{~mL}$ water. For dissolution experiments, PLLA containers are bonded to PLLA layer instead of sacrificial water soluble PAA-PEG layer in order to avoid release of the microcontainers from the substrate during measurements and interference with the UV probe. The PLLA layer after being loaded with drug-polymer matrix is peeled from $\mathrm{Si}$ substrate and cut into individual samples for each of the six probes of $\mu$ DISS profiler. Each sample is first glued with carbon pad on teflon-coated magnetic stirrer.

\section{References}

[1] D. A. Lavan, T. McGuire, R. Langer, Nat. Biotechnol., 2003, 21(10), 1184.

[2] S. Sant, S. L. Tao, O. Z. Fisher, Q. Xu, N. A. Peppas, A. Khademhosseini, Adv. Drug Deliv. Rev. 2012., 64(6), 496.

[3] H. D. Chirra, L. Shao, N. Ciaccio, C. B. Fox, J. M. Wade, A. Ma, T. A. Desai, Adv. Healthcare Mater. 2014, 3(10), 1648.

[4] L. H. Nielsen, A. Melero, S. S. Keller, J. Jacobsen, T. Garrigues, T. Rades, A. Müllertz, A. Boisen, Int. J. Pharm. 2016, 504(1), 98.

[5] R. S. Shawgo, A. C. R. Grayson, Y. Li, M. J. Cima, Curr. Opin. Solid State Mater. Sci. 2002, 6(4), 329.

[6] S. L. Tao, T. A. Desai, Adv. Mater. 2005, 17(13), 1625.

[7] C. B. Fox, H. D. Chirra, T. A. Desai, Curr. Pharm. Biotechnol. 2014, 15(7), 673.

[8] L. H. Nielsen, J. Nagstrup, S. Gordon, S. S. Keller, J. Østergaard, T. Rades, A. Müllertz, A. Boisen, Biomed. Microdevices, 2015, 17(3), 1.

[9] J. C. Jeong, J. Lee, K. Cho, J. Controlled Release, 2003, 92(3), 249.

[10] Y. Qiu, K. Park, Adv. Drug Delivery Rev. 2012, 64, 49.

[11] M. K. Chun, C. S. Cho, H. K. Choi, J. Controlled Release, 2002, 81(3), 327.

[12] H. D. Chirra, T. A. Desai, Small, 2012, 8(24), 3839. 
[13] A. Ahmed, C. Bonner, T. A. Desai, J. Controlled Release, 2002, 81(3), 291.

[14] R. Kolakovic, T. Viitala, P. Ihalainen, N. Genina, J. Peltonen, N. Sandler, Expert Opin. Drug Deliv. 2013, 10(12), 1711.

[15] P. Marizza, S. S. Keller, A. Müllertz, A. Boisen, J. Controlled Release, 2014, 173, 1.

[16] H. P. Lee, W. H. Ryu, Biofabrication, 2013, 5(2), 025011.

[17] R. Mukhopadhyay, Anal. Chem. 2007, 79(9), 3248.

[18] L. H. Nielsen, S. S. Keller, K. C. Gordon, A. Boisen, T. Rades, A. Müllertz. Eur. J. Pharm. Biopharm. 2012, 81(2), 418.

[19] R. S. Petersen, S. S. Keller, A. Boisen, Lab Chip, 2015, 15(12), 2576.

[20] R. S. Petersen, R. Mahshid, N. K. Andersen, S. S. Keller, H. N. Hansen, A. Boisen, Microelectron. Eng. 2015, 133, 104.

[21] R. S. Petersen, S. S. Keller, O. Hansen, A. Boisen, J. Micromech. Microeng. 2015, 25(5), 055021.

[22] B.E. Rapp, M. Schneider, M. Worgull, Microsys. Technol. 2010, 16(7), 1201.

[23] J. N. Babu, S. Cherukuvada, R. Thakuria, A. Nangia, Cryst. Growth Des. 2010, 10(4), 1979.

[24] M. Baiardo, G. Frisoni, M. Scandola, M. Rimelen, D. Lips, K. Ruffieux, E. Wintermantel, J. Appl. Polym. Sci. 2003, 90(7), 1731.

[25] D. Wu, Y. Zhang, M. Zhang, W. Zhou, Eur. Polym. J. 2008, 44(7), 2171.

[26] V. Iannuccelli, G. Coppi, E. Leo, F. Fontana, M. T. Bernabei, Drug Dev. Ind. Pharm. 2000, 26(6), 595.

[27] L. H. Nielsen, S. Gordon, J. P. Pajander, J. Østergaard, T. Rades, A. Müllertz, Int. J. Pharm. 2013, 457(1), 14.

[28] M. A. Woodruff, D. W. Hutmacher, Prog. Polym. Sci. 2010, 35(10), 1217. 
Figure 1. Hot punching for loading of microcontainers with drug-polymer matrices: A. Drugpolymer loading in SU-8 microcontainers by hot punching on deformable substrate: 1. Fabrication of SU-8 microcontainers on fluorocarbon coating and preparation of the sample by spin coating drug-polymer solution on PDMS coated Si. 2. Hot punching drug-polymer by SU-8 microcontainermold, 3.Demolding of the containers with attached drug-polymer matrices punched out from rest of the film, B. Drug-polymer loading in PLLA microcontainers: 1. Fabrication of PLLA microcontainers bonded on water soluble poly acrylic acid (PAA) - polyethylene glycol (PEG) sacrificial layer and preparation of the sample by spin coating drug-polymer solution directly on Si. 2. Hot punching drug-polymer by PLLA microcontainer-mold, 3.Demolding of the containers with attached drug-polymer matrices punched out from rest of the film and loading of the containers. 


\section{Loading SU-8 microcontainers}

A

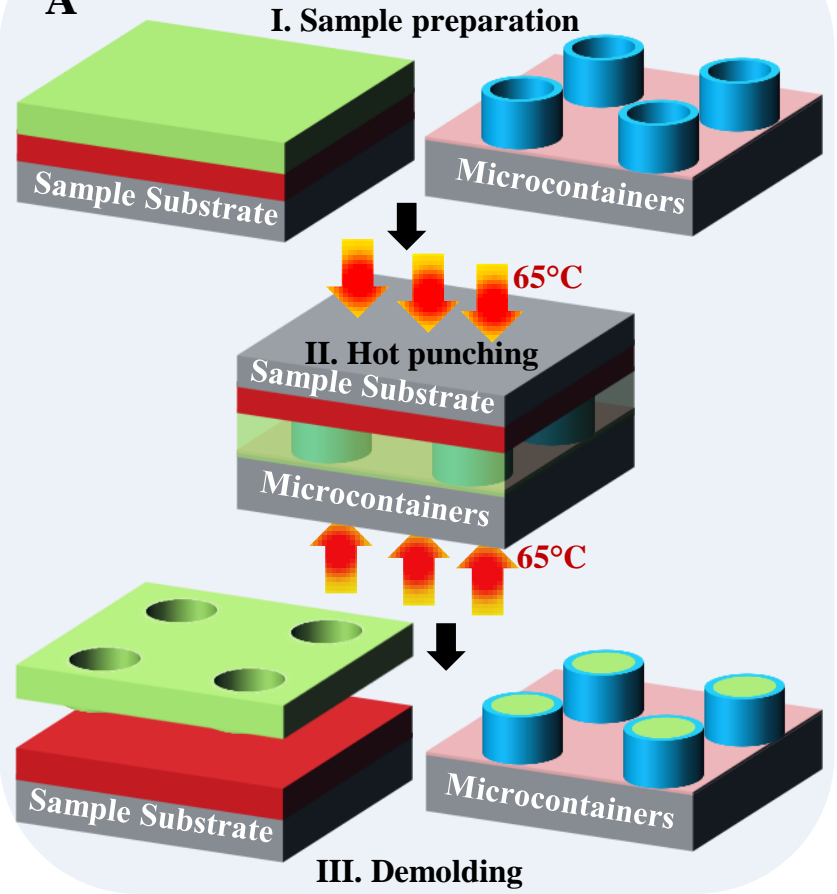

Si substrate
III. Demolding

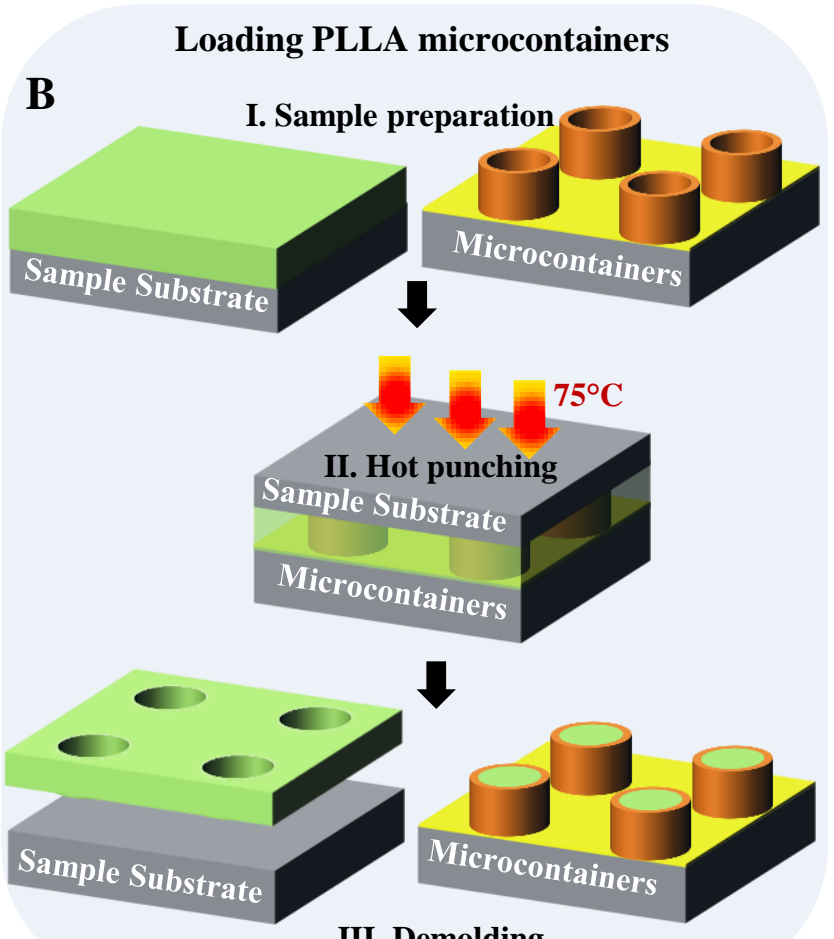

III. Demolding

SU-8 microcontainers Drug-Polymer matrix

PDMS elastic layer

PAA-PEG sacrificial layer

PLLA microcontainers

Figure 2. SEM micrographs of: A. Empty SU-8 microcontainers B/C. F-PCL loading in SU-8 microcontainers using optimized parameters; D.Empty PLLA microcontainers E/F. F-PCL loading in PLLA microcontainers; Optical profiler measurements of G. empty, and H. F-PCL loaded PLLA microcontainers along with the 3D images. 


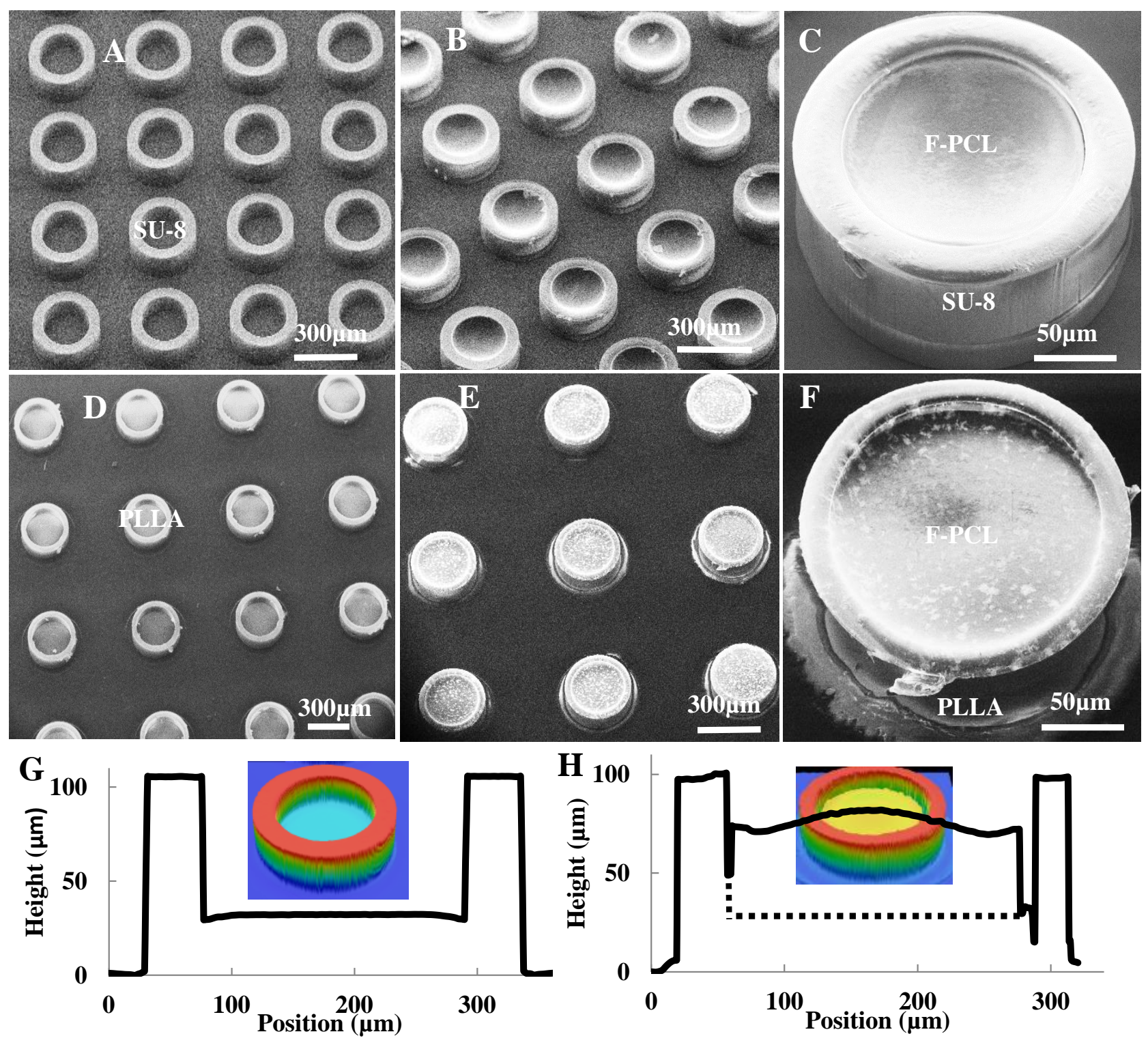

Figure 3. A. Raman Spectra B. X-ray diffractometry of F-PCL matrix loaded in PLLA microcontainers, only PCL polymer loaded in PLLA microcontainers and crystalline furosemide powder. C. Dissolution release of Furosemide from PCL matrix loaded in PLLA microcontainer. 

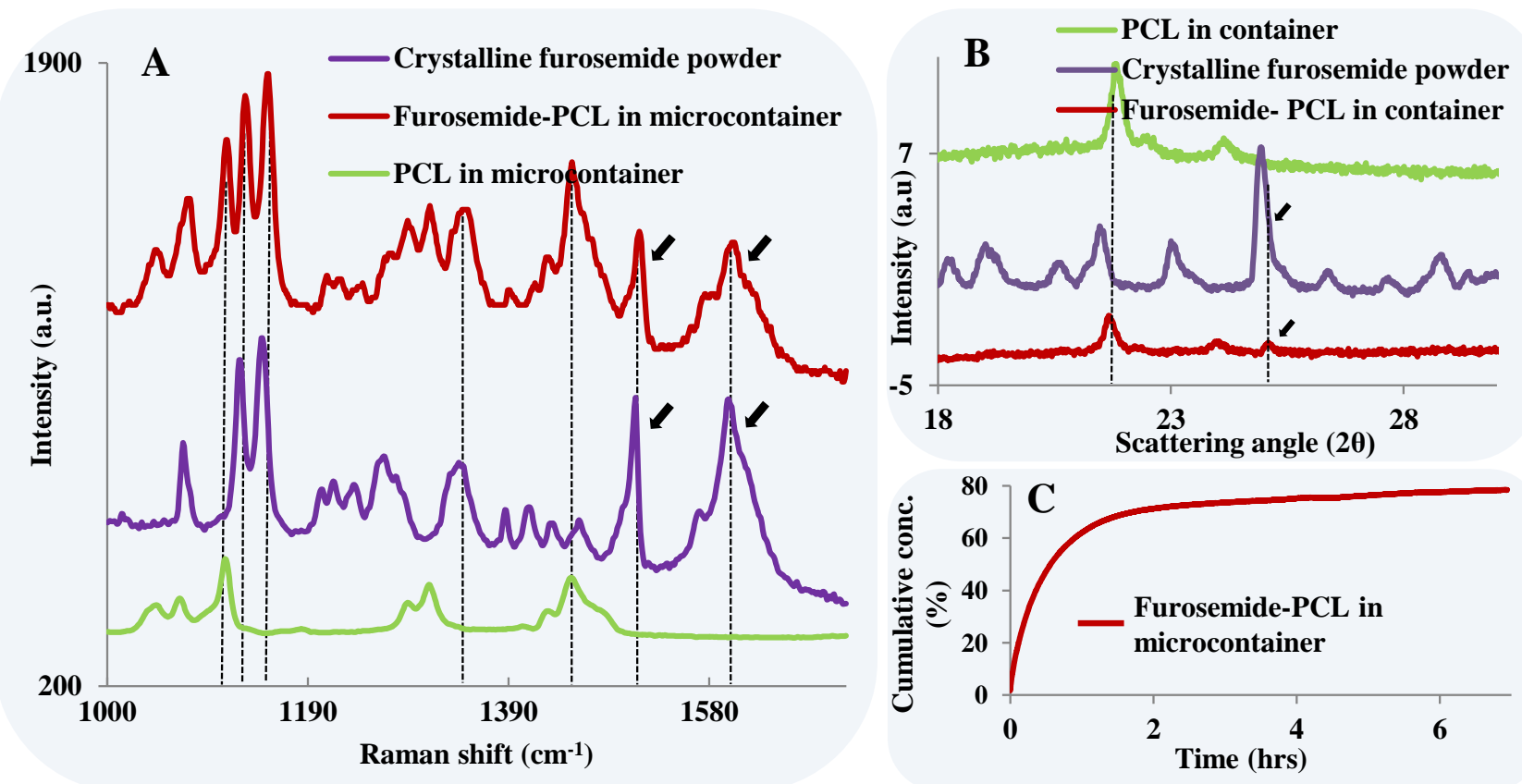

Table 1. Dimensions of SU-8 and PLLA microcontainers 


\begin{tabular}{|c|c|c|c|c|c|}
\hline Container & $\begin{array}{c}\text { Depth of } \\
\text { reservoir, } \\
H_{R}(\mu \mathrm{m})\end{array}$ & $\begin{array}{c}\text { Diameter of } \\
\text { reservoir, } \\
\boldsymbol{D}_{\boldsymbol{R}}(\mu \mathrm{m})\end{array}$ & $\begin{array}{c}\text { Height of } \\
\text { container, } \\
\boldsymbol{H}_{\mu C}(\mu \mathrm{m})\end{array}$ & $\begin{array}{c}\text { Diameter of } \\
\text { container, } \\
\boldsymbol{D}_{\mu C}(\mu \mathrm{m})\end{array}$ & $\begin{array}{c}\text { Center-to-center } \\
\text { distance between } \\
\text { containers, L }(\mu \mathrm{m})\end{array}$ \\
\hline SU-8 & 60 & 200 & 100 & 300 & 450 \\
\hline PLLA & 73 & 240 & 100 & 300 & 600 \\
\hline
\end{tabular}


Calculation of required polymer film thickness

In hot embossing, the total amount of polymer volume $\left(V_{\text {fill }}\right)$ that is required to fill the cavities in the stamp can be calculated assuming that the volume of the polymer is constant. Figure. A is the schematic representation of a typical stamp.

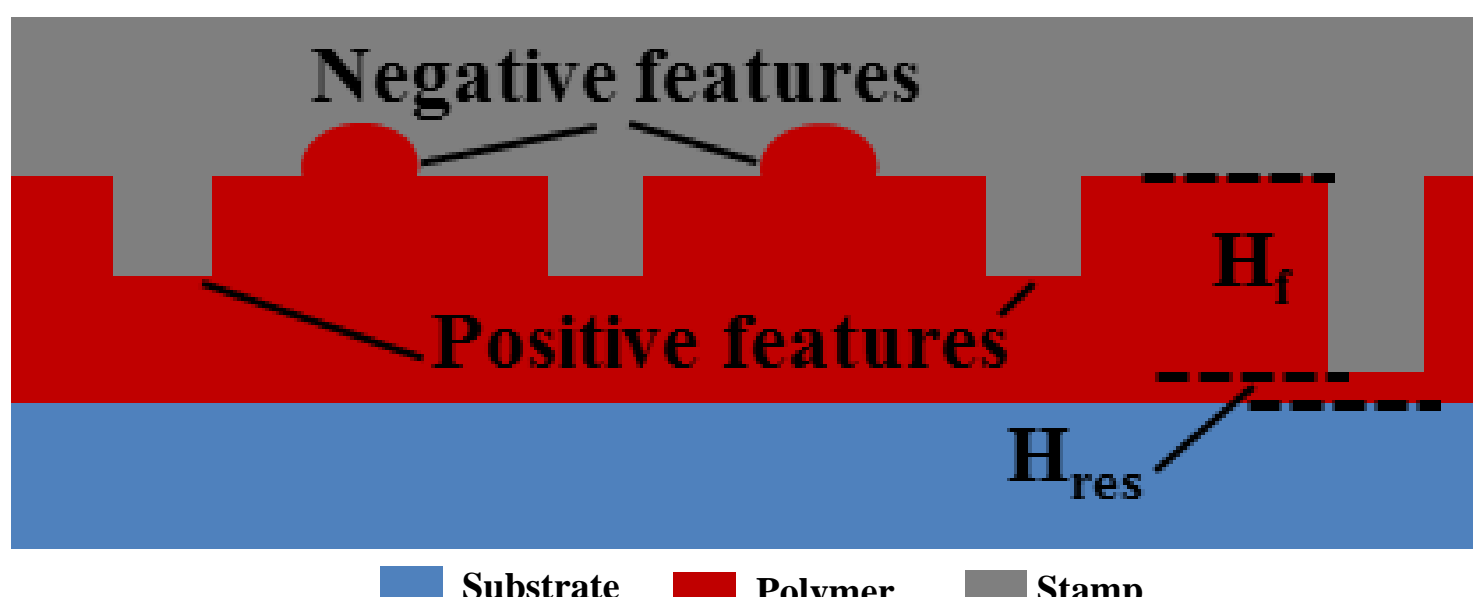

Figure. A. Schematic representation of a typical stamp.

If the highest elevated feature on the stamp has a height, $H_{f}$, the total surface area to be filled on the stamp is $A$, the volume of a negative feature is $V_{\text {neg }}$ and the volume of a positive feature is $V_{\text {pos }}$.

$$
V_{f i l l}=A H_{f}+\sum V_{n e g}-\sum V_{p o s}
$$

If the volume of the residual layer with thickness $H_{\text {res }}$ is taken into account, then the volume $V_{0}$ of the initial polymer layer is:

$$
V_{0}=A H_{\text {res }}+V_{\text {fill }}
$$

This implies that the initial thickness, $H_{0}$ of the polymer that has to be spin coated to achieve complete filling of the mold is:

$$
H_{0}=H_{\text {res }}+\frac{V_{\text {fill }}}{A}
$$


In hot punching, the thickness of the residual layer is assumed to be $H_{r e s}=0$. This means that $H_{0}$ can be derived by the total volume of the cavities. To be able to successfully disrupt the residual layer, the thickness of the spin coated film should be slightly lower than $H_{0}$. In that case polymer
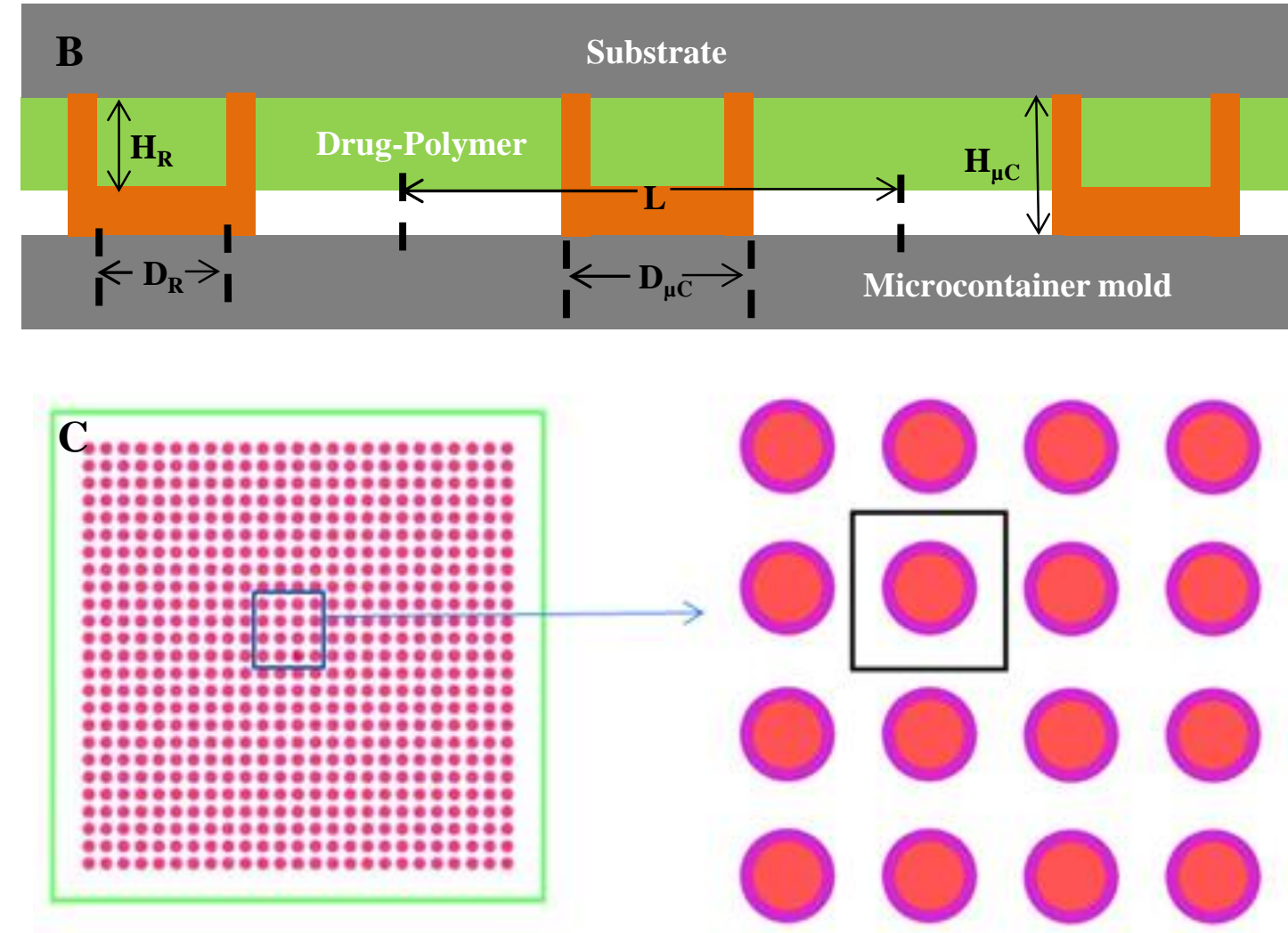

Figure. B. Schematic of hot punching of drug-polymer matrix by microcontainer-mold; C. Top view of the microcontainer chip

fluid can be completely confined in cavities of the mold and the highest protrusions of the stamp can achieve conformal contact with the substrate below the polymer. This thickness is the maximum thickness, $T_{\max }$ of polymer film for hot punching. From Figure. B and C, and Table 1, A. assuming that the microreservoirs of the containers are completely filled, that is the drug-polymer matrix is filled to $H_{R}$, 


$$
L^{2} T_{\max }=L^{2} H_{\mathrm{R}}-\pi H_{R}\left(\frac{D_{\mu \mathrm{C}}^{2}-D_{\mathrm{R}}^{2}}{4}\right)
$$

This implies that the maximum thickness $\left(T_{\max }\right)$ of the F-PCL films required is given by:

$$
T_{\max }=H_{\mathrm{R}}\left\{1-\frac{\pi}{4}\left(\frac{D_{\mu \mathrm{C}}^{2}-D_{\mathrm{R}}^{2}}{L^{2}}\right)\right\}
$$

